Fehlimplantationen im Hinblick auf Achsausrichtung und Rotation, aber auch Notching und anteriorem „Overstuffing " zu vermeiden. Allerdings gibt es wesentliche Designunterschiede der verschiedenen Hersteller, die beachtet werden müssen. Gerade für kleinere Häuser, in denen die Anschaffung eines Navigationsgeräts nicht finanzierbar ist, scheinen individuelle Schnittblöcke besonders attraktiv [1].

Die Wiederherstellung der Knieachsen unter Belassung physiologischer „Fehlstellungen" mittels kinematischem Alignment stellt eine interessante Entwicklung mit guten frühfunktionellen Ergebnissen dar. Ob sich dieses Verfahren auch langfristig über bessere Standzeiten durchsetzen wird, bleibt abzuwarten.

Maßgefertigte Teilprothesen sind bei physiologischer Kinematik und erhaltenem Bandapparat ebenfalls eine überzeugende Entwicklung, wobei der klinische Überlegenheitsnachweis durch Studien noch aussteht. Inwieweit der natürliche Bewegungsablauf auch bei Pangonarthrose und kontraktem Kapsel-BandApparat über maßgefertigte Totalendoprothesen rekonstruiert werden kann, muss ebenfalls die Zukunft zeigen.

Literatur unter www.springermedizin.de/ orthopaedie-und-rheuma

\section{PD Dr. Hans Gollwitzer}

ATOS Klinik München

Effnerstr. 38

81925 München

Klinik für Orthopädie und

Sportorthopädie

Klinikum rechts der Isar der TU München E-Mail: hans.gollwitzer@atos-muenchen.de Internet: www.drgollwitzer.de

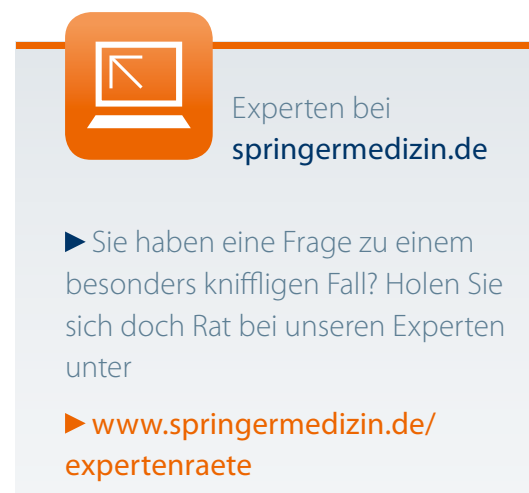

\section{Normalbefunde in der Skelettreifung}

— Dieser einzigartige Atlas mit 2.300 Abbildungen in qualitativ hochwertigster Ausstattung ermöglicht den sofortigen Zugriff auf die Normalbefunde des Skeletts in jedem Entwicklungsabschnitt. Es finden sich mehrere Ansichten für jede Kombination aus Alter, Geschlecht und Körperteil. So werden Referenzpunkte für eine faktenbasierte klinische Beurteilung des untersuchten Falls in hervorragender Weise geliefert. Geordnet nach Geschlecht und Körperteil ist ein schneller Zugriff auf die Bilder der normalen Entwicklung in jedem gewünschten Alter gewährleistet. Die beiliegende DVD enthält alle Abbildungen aus dem Buch, darüber hinaus ermöglicht sie eine virtuelle Skelettbesichtigung in jedem gewünschten Alter. Diese einzigartige Wissensquelle stellt jedem Orthopäden, Radiologen, Kinderarzt und Unfallchirurgen die volle Bandbreite der Vergleichsinformationen zur Verfügung.

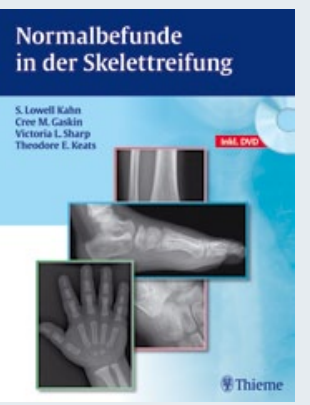

Fazit: Das Buch sollte in keiner Bibliothek fehlen. Der Preis ist der exzellenten Ausstattung mehr als angemessen.

Prof. Klaus. M. Milachowski

S. Lowell Kahn, Cree : Gaskin, Victoria L. Sharp,

Theodore E. Keats

Normalbefunde in der Skelettreifung. Georg Thieme

Verlag 2012, 608 S., 2304 Abb., gebunden, 199,99€ (inkl.

Mwst.); ISBN-10: 3131723416, ISBN-13: 978-3131723413

\title{
FBL Klein-Vogelbach Functional Kinetics praktisch angewandt
}

— Zentrales Anliegen der Autoren der Buchreihe FBL Functional Kinetics ist es, Therapeuten das funktionelle Denken und Handeln nahezubringen. In Band II, der sich mit den Körperabschnitten Brustkorb, Kopf und Arme beschäftigt, diente den Autoren erneut die International Classification of Functioning (ICF) als Grundlage: Aktivität und Partizipation sind Ausgangspunkt der Diagnostik und Ziel der Behandlung; strukturelle Probleme und Funktionsstörungen der Organe liefern Hintergrundinformationen zur funktionellen Störung des Patienten.

Dass Buch vermittelt, wie man Patienten mit Functional Kinetics nach Klein-Vogelbach problemorientiert untersuchen kann. Standardisierte Aktivitätstests erlauben die Beurteilung der Bewegungsqualität und -kontrolle und offenbaren typische Bewegungsstörungen. Es beinhaltet zudem ein detailliertes Trainingsprogramm für die Arbeit am und mit dem Patienten und umfassende praktische Anwendungskonzepte für die Körperabschnitte Brustkorb, Arme und Kopf. Bewegungsanalyse, therapeutische Übungen, Behandlungstechniken, Ballübungen, Atemtech-

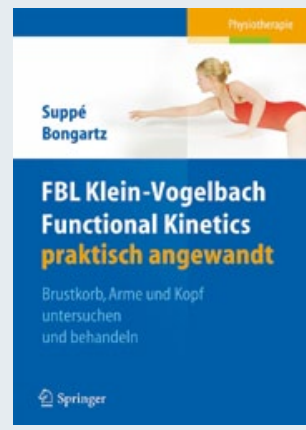
niken - die FBL bietet viele Ideen und Maßnahmen zur individuellen Behandlung von Patienten. Dr. Brigitta Schneider

B. Suppé, M. Bongartz

FBL Klein-Vogelbach Functional Kinetics praktisch angewandt

Brustkorb, Arme und Kopf untersuchen und behandeln; Springer Verlag 2013; X, 98 S., 182 Abb., 173 in Farbe; Formate: Softcover (ISBN 978-3-642-20725-9; 32,95€), eBook (ISBN 978-3-642-20726-6; 24,99€) 